\title{
A high concentration of genistein down-regulates activin A, Smad3 and other TGF- $\beta$ pathway genes in human uterine leiomyoma cells
}

\author{
Xudong Di ${ }^{1,3}$, Danica M.K. Andrews ${ }^{2}$, \\ Charles J. Tucker ${ }^{2}$, Linda $\mathrm{Yu}^{1}$, \\ Alicia B. Moore ${ }^{1}$, Xiaolin Zheng ${ }^{1}$, \\ Lysandra Castro', Tonia Hermon', \\ Hang $\mathrm{Xiao}^{4}$ and Darlene Dixon ${ }^{1,5}$ \\ ${ }^{1}$ Molecular Pathogenesis Group \\ National Toxicology Program (NTP) Laboratories Branch, NTP \\ ${ }^{2}$ Microarray Group \\ Laboratory of Molecular Toxicology \\ National Institute of Environmental Health Sciences \\ National Institutes of Health (NIH) \\ Department of Health and Human Services (DHHS) \\ Research Triangle Park \\ North Carolina 27709, USA \\ ${ }^{3}$ Bureau of Health of Wujin District \\ Changzhou 213159, China \\ ${ }^{4}$ Department of Toxicology \\ School of Public Health \\ Nanjing Medical University \\ Nanjing 210029, China \\ ${ }^{5}$ Corresponding author: Tel, 1-919-541-3814; \\ Fax, 1-919-541-7666; E-mail, dixon@ niehs.nih.gov \\ http://dx.doi.org/10.3858/emm.2012.44.4.024
}

Accepted 7 November 2011

Available Online 6 January 2012

Abbreviations: GnRH, gonadotropin-releasing hormone; SERMs, selective estrogen-receptor modulators; SPRMs, selective progesterone-receptor modulators; UtLM cell, uterine leiomyoma cell

\begin{abstract}
Previously, we found that high doses of genistein show an inhibitory effect on uterine leiomyoma (UtLM) cell proliferation. In this study, using microarray analysis and Ingenuity Pathways Analysis ${ }^{\mathrm{TM}}$, we identified genes (up- or down-regulated, $\geq 1.5$ fold, $P \leq 0.001$ ), functions and signaling pathways that were altered following treatment with an inhibitory concentration of genistein $(50 \mu \mathrm{g} / \mathrm{ml})$ in UtLM cells. Downregulation of
\end{abstract}

TGF- $\beta$ signaling pathway genes, activin A, activin B, Smad3, TGF- $\beta 2$ and genes related to cell cycle regulation, with the exception of the upregulation of the CDK inhibitor P15, were identified and validated by real-time RT-PCR studies. Western blot analysis further demonstrated decreased protein expression of activin A and Smad3 in genistein-treated UtLM cells. Moreover, we found that activin A stimulated the growth of UtLM cells, and the inhibitory effect of genistein was partially abrogated in the presence of activin A. Overexpression of activin A and $S \operatorname{mad} 3$ were found in tissue samples of leiomyoma compared to matched myometrium, supporting the contribution of activin A and Smad3 in promoting the growth of UtLM cells. Taken together, these results suggest that downregulation of activin A and Smad3, both members of the TGF- $\beta$ pathway, may offer a mechanistic explanation for the inhibitory effect of a high-dose of genistein on UtLM cells, and might be potential therapeutic targets for treatment of clinical cases of uterine leiomyomas.

Keywords: activin A; genistein; leiomyoma; myometrium; oligonucleotide array sequence analysis; Smad3 protein; transforming growth factor $\beta$

\section{Introduction}

Uterine leiomyomas (i.e., fibroids, myomas) are the leading indication for hysterectomy in the United States with the estimated cumulative incidence of tumors by age 50 being $>80 \%$ for black women and nearly $70 \%$ for white women in the United States (Walker and Stewart, 2005; Viswanathan et al., 2007). These tumors represent a tremendous public health burden and economic cost to society. At present, surgical intervention is still the standard and most effective treatment for uterine leiomyomas (Rackow and Arici, 2006; Tulandi, 2007). Although therapeutic agents, such as gonadotropin-releasing hormone $(\mathrm{GnRH})$ agonists are being used clinically (Chegini et al., 2002), and selective estrogen-receptor modulators (SERMs) (Liu et al., 2007), selective progesterone-receptor modulators (SPRMs) (Chwalisz et 
Table 1. Functions and genes ${ }^{a}$ that were significantly altered in uterine leiomyoma (UtLM) cells following genistein $(50 \mu \mathrm{g} / \mathrm{ml})$ treatment for $24 \mathrm{~h}$

\begin{tabular}{|c|c|c|c|c|}
\hline Gene function & Genes & Accession No. & Description & Fold change \\
\hline \multicolumn{5}{|l|}{ Cancer } \\
\hline & C5ORF13 & NM_004772 & Chromosome 5 open reading frame 13 & -4.45 \\
\hline & CASP3 & NM_004346 & Caspase 3 , apoptosis-related cysteine protease & -1.65 \\
\hline & CAV1 & BC006432 & Caveolin 1 , caveolae protein, $22 \mathrm{kDa}$ & -2.84 \\
\hline & CLG & AK026111 & Likely ortholog of mouse common-site lymphoma/leukemia GEF & -1.97 \\
\hline & CNN2 & NM_004368 & Calponin 2 & -1.9 \\
\hline & CST & NM_004861 & Galactose-3-O-sulfotransferase 1 & 3.7 \\
\hline & CYR61 & Z97068 & Cysteine-rich, angiogenic inducer, 61 & -2.47 \\
\hline & ENC1 & AY049781 & Ectodermal-neural cortex (with BTB-like domain) & -2.72 \\
\hline & FGF5 & M37825 & Fibroblast growth factor 5 & -2.14 \\
\hline & FGF13 & NM_004114 & Fibroblast growth factor 13 & -2.06 \\
\hline & FGFR3 & NM_000142 & $\begin{array}{l}\text { Fibroblast growth factor receptor } 3 \text { (achondroplasia, thanatophoric } \\
\text { dwarfism) }\end{array}$ & 1.85 \\
\hline & HAS2 & NM_005328 & Hyaluronan synthase 2 & -4.88 \\
\hline & HMGA2 & NM_003483 & High mobility group AT-hook 2 & -3.06 \\
\hline & HMOX1 & NM_002133 & Heme oxygenase (decycling) 1 & 4.99 \\
\hline & ID1 & NM_002165 & Inhibitor of DNA binding 1, dominant negative helix-loop-helix protein & 1.67 \\
\hline & ID3 & NM_002167 & Inhibitor of DNA binding 3, dominant negative helix-loop-helix protein & 1.54 \\
\hline & ID4 & U16153 & Inhibitor of DNA binding 4, dominant negative helix-loop-helix protein & 2.68 \\
\hline & IGF2R & NM_000876 & Insulin-like growth factor 2 receptor & 1.89 \\
\hline & IRS1 & NM_005544 & Insulin receptor substrate 1 & 1.6 \\
\hline & IRS2 & AF073310 & Insulin receptor substrate 2 & 7.88 \\
\hline & MMP1 & NM_002421 & Matrix metalloproteinase 1 (interstitial collagenase) & -2.26 \\
\hline & MMP14 & NM_004995 & Matrix metalloproteinase 14 (membrane-inserted) & 1.88 \\
\hline & RARB & NM_000965 & Retinoic acid receptor, beta & -1.84 \\
\hline & $\mathrm{SHC} 1$ & NM_003029 & SHC (Src homology 2 domain containing) transforming protein 1 & -1.77 \\
\hline & TGFB2 & AK021874 & Transforming growth factor, beta 2 & -3.03 \\
\hline & TGFBR3 & NM_003243 & Transforming growth factor, beta receptor III (betaglycan, 300kDa) & 1.52 \\
\hline & VEGF & AK098750 & Vascular endothelial growth factor & -1.6 \\
\hline & VEGFC & NM_005429 & Vascular endothelial growth factor $\mathrm{C}$ & -2.92 \\
\hline \multicolumn{5}{|l|}{ Cell cycle } \\
\hline & MYBL1 & X66087 & v-myb myeloblastosis viral oncogene homolog (avian)-like 1 & -3.41 \\
\hline & PDGFRA & NM_006206 & Platelet-derived growth factor receptor, alpha polypeptide & -2.63 \\
\hline & HGF & X16323 & Hepatocyte growth factor (hepapoietin A; scatter factor) & -2.32 \\
\hline & $\mathrm{SHC} 1$ & NM_003029 & SHC (Src homology 2 domain containing) transforming protein 1 & -1.77 \\
\hline & RAD21 & NM_006265 & RAD21 homolog (S. pombe) & -1.69 \\
\hline & CASP3 & NM_004346 & Caspase 3 , apoptosis-related cysteine protease & -1.65 \\
\hline & VEGF & AK098750 & Vascular endothelial growth factor & -1.6 \\
\hline & IRS1 & NM_005544 & Insulin receptor substrate 1 & 1.6 \\
\hline & PLK3 & NM_004073 & Polo-like kinase 3 (Drosophila) & 2.25 \\
\hline & PCNA & NM_002592 & Proliferating cell nuclear antigen & 2.36 \\
\hline & IL6R & $\mathrm{X} 12830$ & Interleukin 6 receptor & 3.11 \\
\hline & HMOX1 & NM_002133 & Heme oxygenase (decycling) 1 & 4.99 \\
\hline & PLAB & NM_004864 & Growth differentiation factor 15 & 7.57 \\
\hline & IRS2 & AF073310 & Insulin receptor substrate 2 & 7.88 \\
\hline \multicolumn{5}{|l|}{ Cell death } \\
\hline & EGR1 & NM_001964 & Early growth response 1 & -6.76 \\
\hline & MYBL1 & X66087 & v-myb myeloblastosis viral oncogene homolog (avian)-like 1 & -3.41 \\
\hline & PDGFRB & NM_002609 & Platelet-derived growth factor receptor, beta polypeptide & -3.25 \\
\hline & TGFB2 & AK021874 & Transforming growth factor, beta 2 & -3.03 \\
\hline & VEGFC & NM_005429 & Vascular endothelial growth factor $\mathrm{C}$ & -2.92 \\
\hline & CDK6 & BC027989 & Cyclin-dependent kinase 6 & -2.91 \\
\hline & $\mathrm{SHC} 3$ & NM_016848 & Src homology 2 domain containing transforming protein $\mathrm{C} 3$ & -2.35 \\
\hline & MMP1 & NM_002421 & Matrix metalloproteinase 1 (interstitial collagenase) & -2.26 \\
\hline
\end{tabular}


Table 1. Continued

\begin{tabular}{|c|c|c|c|c|}
\hline Gene function & Genes & Accession No. & Description & Fold change \\
\hline & FGF5 & M37825 & Fibroblast growth factor 5 & -2.14 \\
\hline & CDK5 & NM_004935 & Cyclin-dependent kinase 5 & -1.87 \\
\hline & $\mathrm{SHC1}$ & NM_003029 & SHC (Src homology 2 domain containing) transforming protein 1 & -1.77 \\
\hline & PCNA & NM_002592 & Proliferating cell nuclear antigen & 2.36 \\
\hline & IRS2 & AF073310 & Insulin receptor substrate 2 & 7.88 \\
\hline \multicolumn{5}{|l|}{ Cell signaling } \\
\hline & IRS1 & NM_005544 & Insulin receptor substrate 1 & 1.6 \\
\hline & OXTR & NM_000916 & Oxytocin receptor & -3.32 \\
\hline & TRPV1 & NM_080706 & Transient receptor potential cation channel, subfamily V, member 1 & 2.56 \\
\hline \multicolumn{5}{|c|}{ Cell-to-cell signaling and interaction } \\
\hline & ESM1 & NM_007036 & Endothelial cell-specific molecule 1 & -8.99 \\
\hline & CASP3 & NM_004346 & Caspase 3 , apoptosis-related cysteine protease & -1.65 \\
\hline & VEGF & AK098750 & Vascular endothelial growth factor & -1.6 \\
\hline & BSN & NM_003458 & Bassoon (presynaptic cytomatrix protein) & 5.32 \\
\hline & IRS2 & AF073310 & Insulin receptor substrate 2 & 7.88 \\
\hline \multicolumn{5}{|c|}{ Cellular growth and proliferation } \\
\hline & IRS2 & AF073310 & Insulin receptor substrate 2 & 7.88 \\
\hline & IL6R & $\mathrm{X} 12830$ & Interleukin 6 receptor & 3.11 \\
\hline & CDKN1C & NM_000076 & Cyclin-dependent kinase inhibitor 1C (p57, Kip2) & 2.36 \\
\hline & PCNA & NM_002592 & Proliferating cell nuclear antigen & 2.36 \\
\hline & IGF2R & NM_000876 & Insulin-like growth factor 2 receptor & 1.89 \\
\hline & FGFR3 & NM_000142 & $\begin{array}{l}\text { Fibroblast growth factor receptor } 3 \text { (achondroplasia, thanatophoric } \\
\text { dwarfism) }\end{array}$ & 1.85 \\
\hline & IRS1 & NM_005544 & Insulin receptor substrate 1 & 1.6 \\
\hline & VEGF & AK098750 & Vascular endothelial growth factor & -1.6 \\
\hline & CASP3 & NM_004346 & Caspase 3 , apoptosis-related cysteine protease & -1.65 \\
\hline & DLG7 & NM_014750 & Discs, large homolog 7 (Drosophila) & -1.94 \\
\hline & EGR1 & NM_001964 & Early growth response 1 & -6.76 \\
\hline & PHLDA1 & $\mathrm{BC} 037430$ & Pleckstrin homology-like domain, family A, member 1 & -13.74 \\
\hline
\end{tabular}

Identified by analysis using Ingenuity Pathway Analysis software.

al., 2005), anti-fibrotic agents and growth factor antagonists (Rackow and Arici, 2006) were assessed experimentally for the treatment of these tumors, there is still a paucity of effective nonsurgical therapies. Therefore, searching for potential novel targets for fibroids treatment is a major goal in this field.

Recently, microarray analysis has provided a novel means of elucidating the mechanisms of fibroid development (Leppert et al., 2006; Pan et al., 2007; Litovkin et al., 2008). For example, gene microarray was used to characterize the molecular environment of leiomyomas and matched myometrium during growth, as well as gene expression profiles of leiomyoma and myometrial-derived smooth muscle cells in response to (TGF- $\beta$ ) and GnRH (Levens et al., 2005). Numerous genes have been found to be differentially expressed in leiomyoma and myometrial tissues (Litovkin et al., 2008). Moreover, based on the results of microarray studies, much emphasis has been placed on the roles of the extracellular matrix (ECM), TGF- $\beta$ and collagen composition in fibroid development (Leppert et al., 2006).

Genistein, the major isoflavone in soy, has been shown in vivo to be beneficial in the prevention of a wide variety of chronic diseases, including cancer (Gupta et al., 2010). Furthermore, at high doses ( $\geq$ $25 \mu \mathrm{M}$ ) that can be reached in cell culture models, genistein shows multidirectional actions, such as inhibition of tyrosine kinase and DNA topoisomerase activities, synthesis and release of TGF- $\beta$ and increased apoptosis in the live cell (Polkowski and Mazurek, 2000). In an earlier study, we found that high doses of genistein $(\geq 10 \mu \mathrm{g} / \mathrm{ml})$ had an inhibitory effect on uterine leiomyoma (UtLM) cells (Moore et al., 2007). Because increased cell proliferation is believed to be the most significant contributor to the growth of uterine leiomyomas (Dixon et al., 2002; Leppert et al., 2006), we thought that microarray analysis of the inhibitory effect of a high-dose of genistein $(50 \mu \mathrm{g} / \mathrm{ml})$ on UtLM cells might offer insight on genes and pathways that may be important in arresting the growth of UtLM cells. These data would also provide novel ideas to better 
Table 2. Signaling pathways and genes ${ }^{a}$ that were significantly altered in uterine leiomyoma (UtLM) cells following genistein $(50 \mu \mathrm{g} / \mathrm{ml})$ treatment for 24 h. Genes, CDKN2B, CCNB2, MADH3, MYBL1, INHBA (activin A), INHBB (activin B) and TGFB2 are also named as P15, Cyclin B2, Smad3, A-myb, Activin A, Activin B and TGF- $\beta 2$

\begin{tabular}{|c|c|c|c|c|}
\hline Signaling pathways & Genes & Accession No. & Description & Fold change \\
\hline \multicolumn{5}{|c|}{ Apoptosis signaling pathway } \\
\hline & $\mathrm{BID}$ & NM_001196 & $\mathrm{BH} 3$ interacting domain death agonist & -1.86 \\
\hline & CASP3 & NM_004346 & Caspase 3, apoptosis-related cysteine protease & -1.65 \\
\hline & MAP3K14 & NM_003954 & Mitogen-activated protein kinase kinase kinase 14 & -1.67 \\
\hline & TNFRSF6 & NM_000043 & Tumor necrosis factor receptor superfamily, member 6 & -1.62 \\
\hline \multicolumn{5}{|c|}{ Cell cycle G1S checkpoint regulation pathway } \\
\hline & CDK6 & BC027989 & Cyclin-dependent kinase 6 & -2.91 \\
\hline & CDKN2B & NM_078487 & Cyclin-dependent kinase inhibitor 2B ( $\mathrm{p} 15$, inhibits CDK4) & 2.2 \\
\hline & E2F4 & $\mathrm{BC} 033180$ & E2F transcription factor 4, p107/p130-binding & -1.61 \\
\hline & MADH3 & U68019 & SMAD, mothers against DPP homolog 3 (Drosophila) & -1.78 \\
\hline & TGFB2 & AK021874 & Transforming growth factor, beta 2 & -3.03 \\
\hline & MYBL1 & X66087 & v-myb myeloblastosis viral oncogene homolog (avian)-like 1 & -3.41 \\
\hline \multicolumn{5}{|c|}{ Cell cycle G2M DNA damage checkpoint regulation pathway } \\
\hline & CCNB2 & NM_004701 & Cyclin B2 & -1.96 \\
\hline & YWHAE & U28936 & $\begin{array}{l}\text { Tyrosine 3-monooxygenase/tryptophan 5-monooxygenase } \\
\text { activation protein, epsilon polypeptide }\end{array}$ & -1.62 \\
\hline \multicolumn{5}{|c|}{ Death receptor signaling pathway } \\
\hline & BID & NM_001196 & $\mathrm{BH} 3$ interacting domain death agonist & -1.86 \\
\hline & CASP3 & NM_004346 & Caspase 3 , apoptosis-related cysteine protease & -1.65 \\
\hline & MAP3K14 & NM_003954 & Mitogen-activated protein kinase kinase kinase 14 & -1.67 \\
\hline & TNFRSF6 & NM_000043 & Tumor necrosis factor receptor superfamily, member 6 & -1.62 \\
\hline \multicolumn{5}{|c|}{ ERK MAPK signaling pathway } \\
\hline & DUSP6 & NM_001946 & Dual specificity phosphatase 6 & -2.14 \\
\hline & EIF4E & NM_001968 & Eukaryotic translation initiation factor $4 \mathrm{E}$ & -1.61 \\
\hline & MAPKAPK5 & NM_139078 & Mitogen-activated protein kinase-activated protein kinase 5 & -1.89 \\
\hline & MKNK2 & NM_017572 & MAP kinase-interacting serine/threonine kinase 2 & 2.57 \\
\hline & PLA2G4A & NM_024420 & Phospholipase A2, group IVA (cytosolic, calcium-dependent) & -1.7 \\
\hline & PPP1R14B & AF318364 & Protein phosphatase 1, regulatory (inhibitor) subunit 14B & -1.68 \\
\hline & PPP1R3C & NM_005398 & Protein phosphatase 1, regulatory (inhibitor) subunit 3C & -1.69 \\
\hline & PXN & NM_002859 & Paxillin & -1.76 \\
\hline & $\mathrm{SHC} 1$ & NM_003029 & SHC (Src homology 2 domain containing) transforming protein 1 & -1.77 \\
\hline \multicolumn{5}{|c|}{ Fatty acid biosynthesis pathway (Path 2) } \\
\hline & ACAA2 & NM_006111 & $\begin{array}{l}\text { Acetyl-Coenzyme A acyltransferase } 2 \text { (mitochondrial } \\
\text { 3-oxoacyl-Coenzyme A thiolase) }\end{array}$ & -1.66 \\
\hline & FASN & NM_004104 & Fatty acid synthase & 1.72 \\
\hline & $\mathrm{HADH} 2$ & NM_004493 & Hydroxyacyl-Coenzyme A dehydrogenase, type II & -1.62 \\
\hline \multicolumn{5}{|c|}{ Insulin receptor signaling pathwat } \\
\hline & $\mathrm{ARHQ}$ & NM_012249 & Ras homolog gene family, member Q & 1.92 \\
\hline & EIF4E & NM_001968 & Eukaryotic translation initiation factor $4 \mathrm{E}$ & -1.61 \\
\hline & IRS1 & NM_005544 & Insulin receptor substrate 1 & 1.6 \\
\hline & IRS2 & AF073310 & Insulin receptor substrate 2 & 7.88 \\
\hline & PPP1R14B & AF318364 & Protein phosphatase 1, regulatory (inhibitor) subunit 14B & -1.68 \\
\hline & PPP1R3C & NM_005398 & Protein phosphatase 1, regulatory (inhibitor) subunit 3C & -1.69 \\
\hline & $\mathrm{SHC1}$ & NM_003029 & SHC (Src homology 2 domain containing) transforming protein 1 & -1.77 \\
\hline \multicolumn{5}{|c|}{ Integrin signaling pathway } \\
\hline & ACTA2 & NM_001613 & Actin, alpha 2, smooth muscle, aorta & -4.46 \\
\hline & ACTB & NM_001101 & Actin, beta & -1.67 \\
\hline & ACTG1 & NM_001614 & Actin, gamma 1 & -1.83 \\
\hline & ACTG2 & NM_001615 & Actin, gamma 2, smooth muscle, enteric & -4.86 \\
\hline & $\mathrm{ARHQ}$ & NM_012249 & Ras homolog gene family, member $Q$ & 1.92 \\
\hline & ARPC2 & NM_152862 & Actin related protein $2 / 3$ complex, subunit $2,34 \mathrm{kDa}$ & -1.66 \\
\hline & ARPC3 & NM_005719 & Actin related protein $2 / 3$ complex, subunit $3,21 \mathrm{kDa}$ & -1.66 \\
\hline & BCAR3 & NM_003567 & Breast cancer anti-estrogen resistance 3 & -2.26 \\
\hline
\end{tabular}


Table 2. Continued

\begin{tabular}{|c|c|c|c|c|}
\hline Signaling pathways & Genes & Accession No. & Description & Fold change \\
\hline & CAV1 & BC006432 & Caveolin 1, caveolae protein, $22 \mathrm{kDa}$ & -2.84 \\
\hline & COL1A1 & NM_000088 & Collagen, type I, alpha 1 & -3.41 \\
\hline & ILK & NM_004517 & Integrin-linked kinase & -1.57 \\
\hline & ITGA2 & NM_002203 & Integrin, alpha 2 (CD49B, alpha 2 subunit of VLA-2 receptor) & -1.64 \\
\hline & LAMA1 & AK091949 & Laminin, alpha 1 & 3.72 \\
\hline & MYLK & NM_053025 & Myosin, light polypeptide kinase & -3.24 \\
\hline & PARVA & NM_018222 & Parvin, alpha & -1.88 \\
\hline & PPP1R12A & AF086032 & Protein phosphatase 1 , regulatory (inhibitor) subunit $12 \mathrm{~A}$ & -2.38 \\
\hline & PPP1R12B & NM_032105 & Protein phosphatase 1 , regulatory (inhibitor) subunit 12B & -3.98 \\
\hline & PXN & NM_002859 & Paxillin & -1.76 \\
\hline & $\mathrm{SHC1}$ & NM_003029 & SHC (Src homology 2 domain containing) transforming protein 1 & -1.77 \\
\hline & VASP & NM_003370 & Vasodilator-stimulated phosphoprotein & -2.07 \\
\hline \multicolumn{5}{|c|}{ P38 MAPK signaling pathway } \\
\hline & DDIT3 & NM_004083 & DNA-damage-inducible transcript 3 & 2.42 \\
\hline & MAPKAPK5 & NM_139078 & Mitogen-activated protein kinase-activated protein kinase 5 & -1.89 \\
\hline & MKNK2 & NM_017572 & MAP kinase-interacting serine/threonine kinase 2 & 2.57 \\
\hline & PLA2G4A & NM_024420 & Phospholipase A2, group IVA (cytosolic, calcium-dependent) & -1.7 \\
\hline & TGFB2 & AK021874 & Transforming growth factor, beta 2 & -3.03 \\
\hline & TNFRSF6 & NM_000043 & Tumor necrosis factor receptor superfamily, member 6 & -1.62 \\
\hline \multicolumn{5}{|c|}{ Purine metabolism pathway } \\
\hline & ADARB1 & NM_015833 & Adenosine deaminase, RNA-specific, B1 (RED1 homolog rat) & 1.67 \\
\hline & ADCY7 & NM_001114 & Adenylate cyclase 7 & -2.23 \\
\hline & AK2 & NM_172199 & Adenylate kinase 2 & -2.65 \\
\hline & AK5 & BC012467 & Adenylate kinase 5 & -1.9 \\
\hline & ATIC & NM_004044 & $\begin{array}{l}\text { 5-aminoimidazole-4-carboxamide ribonucleotide } \\
\text { formyltransferase/IMP cyclohydrolase }\end{array}$ & -1.59 \\
\hline & DGUOK & NM_080916 & Deoxyguanosine kinase & -1.56 \\
\hline & ENPP1 & NM_006208 & Ectonucleotide pyrophosphatase/phosphodiesterase 1 & 2.13 \\
\hline & HPRT1 & NM_000194 & $\begin{array}{l}\text { Hypoxanthine phosphoribosyltransferase } 1 \text { (Lesch-Nyhan } \\
\text { syndrome) }\end{array}$ & -1.69 \\
\hline & IFNAR1 & NM_000629 & Interferon (alpha, beta and omega) receptor 1 & -2.07 \\
\hline & IMPDH2 & NM_000884 & IMP (inosine monophosphate) dehydrogenase 2 & 1.5 \\
\hline & ITPA & NM_033453 & Inosine triphosphatase (nucleoside triphosphate pyrophosphatase) & -1.67 \\
\hline & NT5C & NM_014595 & 5', 3'-nucleotidase, cytosolic & -1.55 \\
\hline & PAICS & $\times 53793$ & $\begin{array}{l}\text { Phosphoribosylaminoimidazole carboxylase, } \\
\text { phosphoribosylaminoimidazole succinocarboxamide synthetase }\end{array}$ & -1.61 \\
\hline & PAPSS2 & AF074331 & 3'-phosphoadenosine 5'-phosphosulfate synthase 2 & -1.72 \\
\hline & PNPT1 & NM_033109 & Polyribonucleotide nucleotidyltransferase 1 & -1.63 \\
\hline & POLI & NM_007195 & Polymerase (DNA directed) iota & -1.86 \\
\hline & REV3L & NM_002912 & REV3-like, catalytic subunit of DNA polymerase zeta (yeast) & -1.82 \\
\hline & RRM2 & BC030154 & Ribonucleotide reductase M2 polypeptide & -2.9 \\
\hline \multicolumn{5}{|c|}{ TGF- $\beta$ signaling pathway } \\
\hline & BMP2 & NM_001200 & Bone morphogenetic protein 2 & 2.81 \\
\hline & INHBA & AK001903 & Inhibin, beta A (activin A, activin AB alpha polypeptide) & -2.06 \\
\hline & INHBB & NM_002193 & Inhibin, beta B (activin AB beta polypeptide) & -1.64 \\
\hline & MADH3 & U68019 & SMAD, mothers against DPP homolog 3 (Drosophila) & -1.78 \\
\hline & SERPINE1 & NM_000602 & $\begin{array}{l}\text { Serine (or cysteine) proteinase inhibitor, clade } \mathrm{E} \text { (nexin, plasminogen } \\
\text { activator inhibitor type 1), member } 1\end{array}$ & -2.1 \\
\hline & SMURF1 & NM_020429 & E3 ubiquitin ligase SMURF1 & -1.68 \\
\hline & TGFB2 & AK021874 & Transforming growth factor, beta 2 & -3.03 \\
\hline \multicolumn{5}{|c|}{ VEGF signaling pathway } \\
\hline & ACTA2 & NM_001613 & Actin, alpha 2, smooth muscle, aorta & -4.46 \\
\hline & ACTB & NM_001101 & Actin, beta & -1.67 \\
\hline & ACTG1 & NM_001614 & Actin, gamma 1 & -1.83 \\
\hline & ACTG2 & NM_001615 & Actin, gamma 2, smooth muscle, enteric & -4.86 \\
\hline
\end{tabular}


Table 2. Continued

\begin{tabular}{|c|c|c|c|c|}
\hline Signaling pathways & Genes & Accession No. & Description & Fold change \\
\hline & EIF2S2 & NM_003908 & Eukaryotic translation initiation factor 2 , subunit 2 beta, $38 \mathrm{kDa}$ & -1.69 \\
\hline & ELAVL1 & BC003376 & $\begin{array}{l}\text { ELAV (embryonic lethal, abnormal vision, Drosophila)-like } 1 \\
\text { (Hu antigen R) }\end{array}$ & -1.6 \\
\hline & $\mathrm{PXN}$ & NM_002859 & Paxillin & -1.76 \\
\hline & $\mathrm{SHC1}$ & NM_003029 & SHC (Src homology 2 domain containing) transforming protein 1 & -1.77 \\
\hline & VEGF & AK098750 & Vascular endothelial growth factor & -1.6 \\
\hline & VEGFC & NM_005429 & Vascular endothelial growth factor $\mathrm{C}$ & -2.92 \\
\hline & YWHAE & U28936 & $\begin{array}{l}\text { Tyrosine 3-monooxygenase/tryptophan 5-monooxygenase } \\
\text { activation protein, epsilon polypeptide }\end{array}$ & -1.62 \\
\hline
\end{tabular}

${ }^{a}$ Identified by analysis using Ingenuity Pathway Analysis software.

understand the function of how cells and integrated biological mechanisms are involved in UtLM growth inhibition, and possibly provide novel targets for clinical intervention.

\section{Results}

\section{Microarray analysis of significantly altered genes}

The expression profile of over 41,000 genes was studied in genistein-treated UtLM cells (http://www. ncbi.nlm.nih.gov/geo/query/acc.cgi?token=vjcjruqa seesmps\&acc=GSE19477). Vehicle treated UtLM cells served as the reference for the expression profile. Signature genes were generated by Rosetta Resolver based on the criteria that genes were $\geq$ 1.5 -fold and $P \leq 0.001$ throughout all replicates. A total of 541 differentially expressed genes were observed. Of these, 150 genes were up-regulated and 391 genes were down-regulated in genistein-treated compared to vehicle-treated UtLM cells. These genes were considered signature genes and were used for further analysis using IPA.

\section{IPA}

To further analyze the biological significance of signature genes, genes were characterized into networks, functions and signaling pathways by using IPA software according to Ingenuity Pathways Knowledge Base (IPKB, http://www.ingenuity.com). These networks, functions and signaling pathways were ranked according to IPA calculated scores, which is based on the significance of involved genes. Based on IPKB, signature genes were classified into multiple functions and pathways by IPA. Among these, six functions and twelve pathways which were identified by IPA with significance values less than or equal to 0.05 (Tables 1 and 2). In the present study, we selected the following genes, INHBA (activin A), INHBB (activin B), MADH3
(Smad3) and TGF- $\beta_{2}$ involved in TGF- $\beta$ signaling pathway, and genes, CDK6, CDKN2B (P15), MYBL1 (A-myb) and CCNB2 (cyclin B2) in cell cycle regulation for further evaluation. We found that most of all selected genes were down-regulated in the high concentration of genistein treated UtLM cells except the CDK inhibitor, P15, which was up-regulated.

\section{Validation of selected function and signaling pathway genes}

Based on the analysis results from IPA software, the eight selected genes were further validated by real-time RT-PCR. As shown in Figure 1, we found that the results of real-time RT-PCR showed similar patterns to that of the microarray, however, the data calculated from the results of real-time RT-PCR was higher compared to microarray data. For example, the expression of P15 was increased in genistein treated UtLM cells about 2-fold by microarray and 11-fold by real-time RT-PCR, while gene expression of cyclin B2, CDK6 and A-myb displayed downregulation of 1.96, 2.96, 3.41-fold change, respectively, in microarray analysis and showed 5, 3, 3-fold decreased expression, respectively, by using real-time RT-PCR (Figure 1A). For TGF- $\beta$ signaling pathway genes, we found that gene expression of activin $A$, activin $B$, Smad 3 and TGF- $\beta_{2}$ were down-regulated by a 2.06, 1.64, 1.78 and 3.03-fold change, respectively, in microarray analysis, and down-regulated by a 2, 4, 2 and 3-fold change, respectively, by real-time RT-PCR (Figure 1B). The differences in the results has been attributed to differences in RT-PCR vs. microarray methodologies.

\section{Decreased protein expression of activin A and Smad3 in genistein-treated UtLM cells}

Activin $A$, is an active TGF- $\beta$ ligand dimer assembled by two INHBA isoforms (activin $A$ ) which initiates cellular responses by binding to its receptors, that 
A

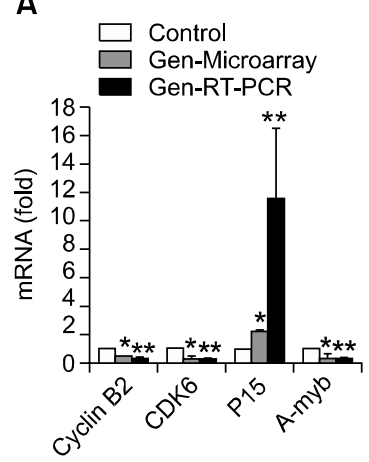

B

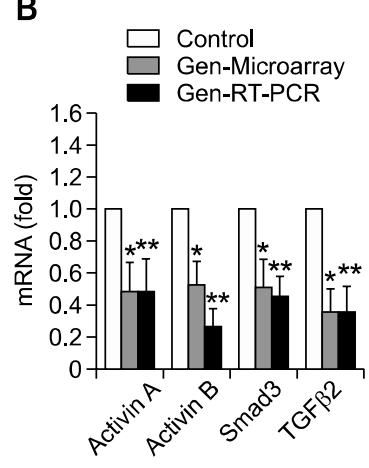

Figure 1. Relative mRNA expression changes of selected pathway genes in uterine leiomyoma (UtLM) cells following genistein $(50 \mu \mathrm{g} / \mathrm{ml})$ treatment for $24 \mathrm{~h}$, as measured by microarray (Gen-Microarray) and real-time RT-PCR (Gen-RT-PCR). Results are calculated as the mRNA fold change by dividing values by the vehicle control (set at 1). Data are presented as mean \pm S.E. of three experiments. A statistically significant difference $(P \leq 0.05)$ is indicated by asterisk(s) $\left(^{*}\right.$ : genistein treated versus vehicle control with the method of microarray; ${ }^{* *}$ : genistein treated versus vehicle control with the method of real-time RT-PCR). A house keeping gene, GAPDH, was used for normalization. (A) Genes related to cell cycle regulation. (B) Genes in TGF- $\beta$ signaling pathway.

A
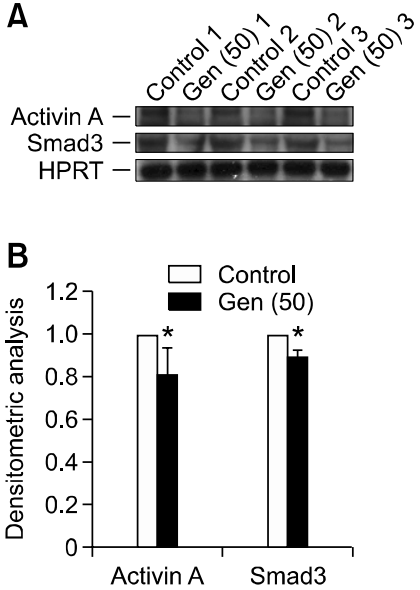

Figure 2. (A) Protein expression of activin $A$ and $S$ mad 3 in uterine leiomyoma (UtLM) cells following genistein $(50 \mu \mathrm{g} / \mathrm{ml})$ treatment for $24 \mathrm{~h}$. Hypoxanthine phosphoribosyl-transferase (HPRT) was used as a protein loading control for each experiment. (B) Bar graphs show the mean \pm $S$.E. values of densitometry data of three experiments by dividing values by the vehicle control (set at 1). A statistically significant difference $(P \leq$ $0.05)$ is indicated by an asterisks (*: genistein treated versus vehicle control).

A
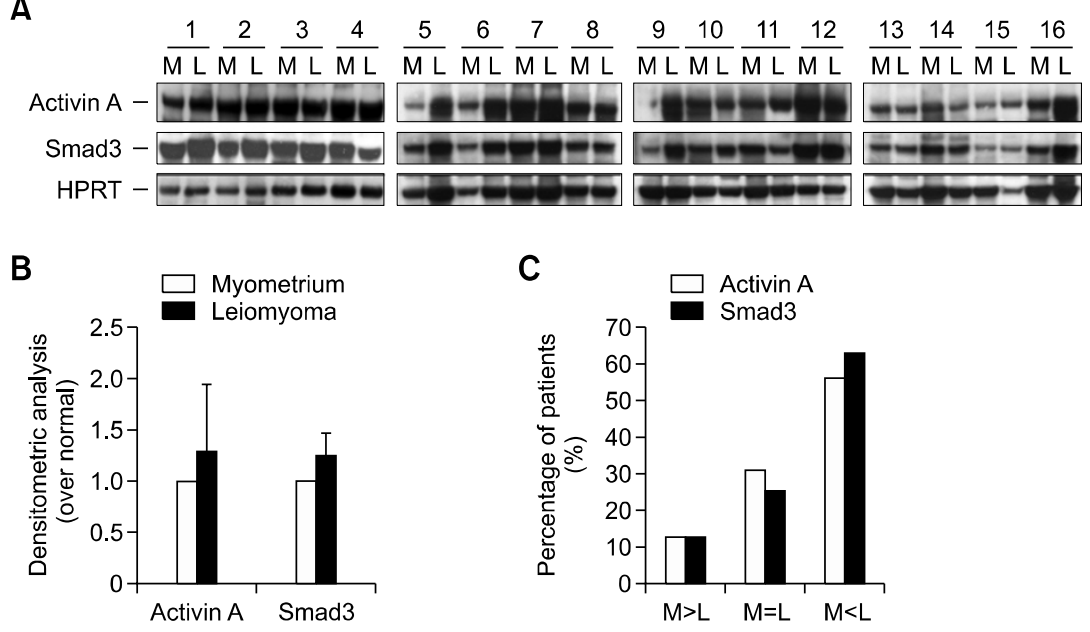

C

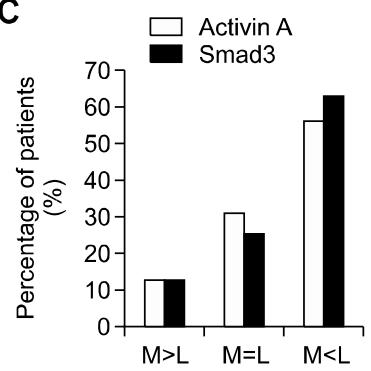

Figure 3. (A) Protein expression of activin $A$ and Smad3 in uterine leiomyomas $(L)$ and patient-matched myometrium (M). Hypoxanthine phosphoribosyl-transferase (HPRT) was used as a protein loading control for each experiment. (B) Bar graphs show the mean \pm S.E. values of densitometry data of three experiments by dividing values by patient-matched myometrium (set at 1). (C) Bar graphs shows the comparison of activin $A$ and Smad3 protein expression between leiomyoma and patient-matched myometrial tissue. have intrinsic serine/threonine kinase activity, and activating downstream Smad proteins (Abe et al., 2004). Because gene and mRNA expression of activin $A$, activin $B$ and Smad3 was down-regulated in genistein-treated UtLM cells, we did further studies to determine if the protein expression of activin A and Smad3 correlated with gene downregulation in genistein-treated UtLM cells. Using western blotting, we found that the protein expression of both activin A and Smad3 was decreased in genistein-treated UtLM cells compared to vehicletreated (Figure 2), suggesting that the decreased protein expression of activin A and Smad3 might due to the down-regulation of INHBA and Smad3 genes, possibly leading to the inhibitory effects of genistein on UtLM cells.

\section{Comparison of activin A and Smad3 protein expression between uterine leiomyomas and matched myometrium in tissue samples}

We examined the protein expression of activin $A$ and Smad3 in sixteen leiomyomas and patient-matched myometrial tissue samples by western blotting to determine whether both of the protein levels of activin $\mathrm{A}$ and Smad3 are overexpressed in uterine leiomyomas compared to the matched myometrial tissue. We found that both protein expression of activin $A$ and Smad3 were detectable in uterine leiomyomas and myometrium (Figure $3 \mathrm{~A}$ ). Although 
A

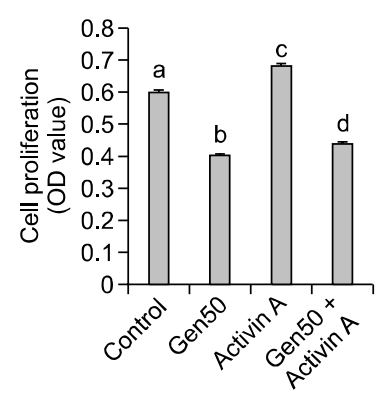

B

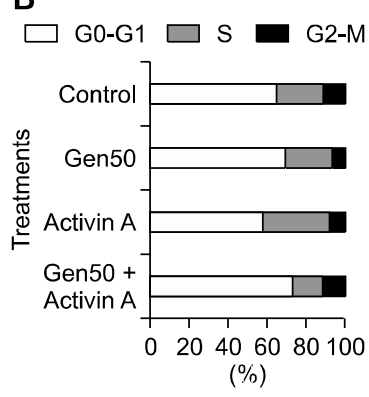

Figure 4. (A) Cell proliferation assay in treated UtLM cells, vehicle (control), $50 \mathrm{mg} / \mathrm{ml}$ of genistein (Gen-50), $20 \mathrm{nM}$ activin A or genistein plus $20 \mathrm{nM}$ activin $\mathrm{A}$ (Gen-50+Acitivin A) for $96 \mathrm{~h}$. The bar graph shows the mean \pm S.E. values of absorbance of three experiments that have been normalized to the vehicle control (set at 1). a, b, $c$ and $d$ indicate groups which are statistically different $(P \leq 0.05)$. (B) Cell cycle analysis in treated UtLM cells, vehicle (control), $50 \mathrm{mg} / \mathrm{ml}$ of genistein (Gen-50), $20 \mathrm{nM}$ activin A or genistein plus $20 \mathrm{nM}$ activin A (Gen-50 + Acitivin A) for $48 \mathrm{~h}$. The values represent the number of cells in the different phases of the cell cycle as a percentage of total cells observed.

no statistical significance was found, in the comparison of uterine leiomyomas and matched myometrial tissue (Figure $3 \mathrm{~B}$ ), activin $\mathrm{A}$ protein levels were higher in leiomyoma than myometrial tissue in $56 \%$ $(9 / 16)$ of the samples; equal in $31 \%(5 / 16)$ of the samples; and lower in $13 \%(2 / 16)$ of the samples (Figure 3C). The protein expression level of Smad3 was higher in $62 \%(10 / 16)$ of the leiomyoma as compared to their matched myometrial tissue; equal in $25 \%(4 / 16)$ of the samples; and lower in leiomyoma than myometrial tissue in $13 \%(2 / 16)$ of the samples. Moreover, a positive correlation was found between the protein expression of activin A and Smad3 in both leiomyomas and matched myometrial tissue (Figures $3 \mathrm{~A}$ and $3 \mathrm{~B}$ ), suggesting that signaling of TGF- $\beta$ pathway, from activin $A$ to Smad3 may have a promotional effect on the growth of leiomyomas.

\section{Activin A stimulates growth of UtLM cells}

In order to gain information on the biological effects of activin A on UtLM cells growth, we utilized a recombinant activin $A$ protein and examined its effects by cell proliferation assays. We found that when UtLM cells were treated with activin $A(20 \mathrm{nM})$ for 5 days, the growth of UtLM cells was stimulated and the number of UtLM cells was significantly higher than vehicle-treated $(P<0.05)$ (Figure 4). Furthermore, when UtLM cells were treated with a high concentration of genistein in combination with activin $A$, we found that the inhibitory effect of genistein was partially antagonized $(P<0.05)$ (Figure 4$)$. Therefore, the results further support the hypothesis

that downregulation of activin A expression by genistein may inhibit the growth of UtLM cells.

\section{Activin A increases percentage of UtLM cells in S phase}

We conducted cell cycle analysis studies using flow cytometry to support our findings of activin $A$ induced increased proliferation in UtLM cells. We found that activin $A$ increased the percentage of cells in $S$ phase in UtLM cells at $48 \mathrm{~h}$ (Figure 4). Additionally, when UtLM cells were treated with a high concentration of genistein in combination with activin $A$, we found the percentage of cells in G2-M increased. These results support the positive growth effects observed in UtLM cells following activin A treatment and the ability of activin A to rescue UtLM cells from the inhibitory growth effects observed with a high concentration of genistien. These data supports the proliferation data and further strengthens the hypothesis that genestein's down regulation of activin A may contribute to its negative growth effect in UtLM cells.

\section{Discussion}

The purpose of the present study was to identify the novel pathways and genes that might be involved in the inhibitory effect of a high dose of genistein on UtLM cells. Using DNA microarray and IPA, we identified genes, functions and pathways that may contribute to the inhibitory effect of genistein on UtLM cells. Most importantly, the possible role of TGF- $\beta$ pathway genes, activin A and Smad 3 in the pathogenesis of human uterine leiomyomas was ascertained.

The growth-inhibitory or apoptosis-inducing effects of genistein at high concentrations are mostly reported in in vitro studies (Jamadar-Shroff et al., 2009; Pavese et al., 2010). By using DNA microarray, specific gene expression patterns have been reported in several different cell types under genistein treatment (Piao et al., 2006; Lee et al., 2007; Lavigne et al., 2008). Consistently, in the present study we found that genes, related to cell cycle progression, such as BID, CASP3, MAP3K14, TNFRSF6, A-myb, CDK6, cyclin B2, Smad3 and E2F4 were significantly downregulated in genisteintreated UtLM cells. We also found that p15, a p53 inducible gene, was induced in UtLM cells. This is consistent with the reported genotoxic/DNA damaging effect of high doses of genistein (Ye et al., 2004) and also is consistent with our previous report of decreased apoptosis in UtLM cells treated with a high dose of genistein compared to uterine myometrial 
(UtSMC) cells (Moore et al., 2007). Herein, our results suggest that regulations of cycle-related genes may be a molecular signature and mechanism of action for high doses of genistein on UtLM cell growth.

In addition to these genes discussed above, we also found that genistein at a high dose down-regulates TGF- $\beta$ signaling pathway genes in UtLM cells. Smad3 is one of two homologous proteins that involved in signaling from TGF-beta/activin to modulate gene transcription (ten Dijke and Hill, 2004). In leiomyoma, the protein expression of Smad3 as well as several other components of the TGF- $\beta$ system, such as TGF $\beta$ receptors and Smad4, was found elevated compared to matched myometrium, and was lowered after GnRHa therapy (Chegini et al., 2003; Levens et al., 2005). Increased expression of Smad3 and TGF- $\beta$ receptors may account for the development of leiomyoma and its matrix components. Therefore, downregulation of Smad3 mRNA expression and decreased protein expression in UtLM cells may contribute to the inhibitory effects of high doses of genistein. Consistent with previous studies, in the present study, we have confirmed that the protein expression of Smad3 is increased in the leiomyomas more than matched myometrial tissue (Chegini et al., 2003). Additionally, we also demonstrated that activin $A$ which is assembled by two INHBA isoforms, was lowered in genistein-treated UtLM cells. Activin $A$, as a member of TGF- $\beta$ family, controls many physiological processes such as cell proliferation and differentiation, immune responses, wound repair and various endocrine activities (Harrison et al., 2005). Herein, we speculate that the inhibition effects of genistein in UtLM cells might be due to the down-regulation of activin A and Smad3, or possibly due to the inhibition of the TGF- $\beta$ signaling, which transducts from activin $A$ to Smad3. Elevation of TGF- $\beta$ s has been reported in uterine leiomyomas (Levens et al., 2005; Luo et al., 2005; Zhao et al., 2007); however, the protein expression of activin $A$ has never been investigated. It was reported that in the ovaries of vertebrates, activins are expressed predominantly in the follicular layer of the oocyte where they regulate processes such as folliculogenesis, steroid hormone production, and oocyte maturation (Hogg, et al., 2010; McLaughlin, et al., 2010). In our present study, the presence of an increased protein expression of activin $A$ in leiomyomas provided further information that activin A may play a role to elevate the growth of uterine leiomyoma. Herein, the results from tissue samples are further supportive of the importance of the TGF- $\beta$ system as key regulator of leiomyoma growth. Activin $A$ has various effects on diverse biological systems (Abe et al., 2004). In contrast to the report that activin $A$ mediates growth inhibition and cell cycle arrest through Smads in human breast cancer cells (Burdette et al., 2005), the stimulatory effect on mesenchymal cells such as fibroblasts has emphasized the importance of activin $A$ in keloid pathogenesis (Mukhopadhyay et al., 2007). In the present study, we found that the growth of UtLM cells was increased by activin A and the inhibitory effect of a high-dose of genistein was antagonized by co-treatment with activin $A$. The stimulatory effect of activin A on UtLM cell growth is similar to TGF- $\beta$, which has been emphasized as one of the promoters in the formation of the extracellular matrix (Leppert et al., 2006). Also, we found that activin A was able to stimulate UtLM cells to progress into the $S$ phase, in addition to promoting the progression of cells into mitosis when administered with a high concentration of genistein. These results further suggest that signaling of TGF- $\beta$ pathway; from activin $A$ to Smad3 may have a promotional effect on the growth of leiomyomas.

In summary, by using IPA, along with multiple altered functions and signaling pathways, we were able to identify that cell cycle dysregulation and down-regulation of the TGF- $\beta$ signaling pathway might contribute to the inhibitory effects of a high dose of genistein in UtLM cells. Using additional complimentary experiments, we were able to show that activin $A$ and Smad3, both members of the TGF- $\beta$ signaling pathway, may offer a mechanistic explanation for the progression of leiomyomas, and that these pathways and genes might be potential therapeutic targets for uterine fibroids.

\section{Methods}

\section{Cell culture}

Human uterine leiomyoma (UtLM) cells (GM10964) were purchased from Coriell Institute for Medical Research (Camden, NJ), and cultured as previously reported (Swartz et al., 2005). Briefly, cells were grown in Eagle's minimum essential medium (Invitrogen [Gibco], Carlsbad, CA) with $19 \%$ fetal bovine serum (FBS) (Sigma, St Louis, MO) and supplemented with vitamins, essential and non-essential amino acids and L-glutamine (Invitrogen). All culture maintenance and experiments were performed at $37^{\circ} \mathrm{C}$ and $5 \%$ $\mathrm{CO}_{2}$. UtLM cells were seeded in T175 flasks (at passages $8-14$ at $80-95 \%$ confluence), and $24 \mathrm{~h}$ prior to treatment, the media was changed to Dulbecco's modified Eagle's medium/nutrient mixture F-12 Ham (DMEM/F-12) (Hyclone Laboratories, Logan, UT) phenol red-free with charcoal dextran treated FBS (Sigma). Cells were then treated with either genistein $(50 \mu \mathrm{g} / \mathrm{ml})(4$ ', 5, 7-Trihydroxyisoflavone; Sigma Chemical Company, St. Louis, MO) or the vehicle control $[0.3 \%$ dimethylsulfoxide (DMSO), Sigma] in phenol red-free, stripped-serum DMEM medium for $24 \mathrm{~h}$. 


\section{RNA isolation and microarray analysis}

Total RNA preparation for microarray studies was performed as previously described (Swartz et al., 2005). Briefly, total RNA was isolated with TRIzol agent (Invitrogen) from treated UtLM cells and purified using a RNeasy MidiKit (QIAGEN, Valencia, CA), according to the manufacturers' protocols. Due to the expense of chip printing and the large amount of RNA needed for each of the hybridizations, the samples from the flasks that received the same treatment were pooled, then concentrated to $>9 \mu \mathrm{g} / \mu \mathrm{l}$ by Microcon 30 columns (Amicon: Millipore, Bedford, MA). Gene expression analysis was conducted using Agilent Whole Human Genome arrays (Agilent Technologies, Palo Alto, CA). Total RNA was amplified by using the Agilent Low RNA Input Fluorescent Linear Amplification Kit protocol. Starting with $500 \mathrm{ng}$ of total RNA, cyanine (Cy) 3- or Cy5-labeled cRNAs were produced according to manufacturer's protocol. For each two-color comparison, $750 \mathrm{ng}$ each of Cy3- and Cy5-labeled cRNAs were mixed and fragmented using the Agilent In Situ Hybridization Kit protocol. Hybridizations were performed for $17 \mathrm{~h}$ in a rotating oven using the Agilent 60-mer Oligo microarray processing protocol. Slides were later washed as indicated in this protocol, and then scanned with an Agilent Scanner. Data were obtained using the Agilent Feature Extraction software (v7.5), employing defaults for all parameters. Since only those genes that were consistently under- or over-expressed on three of four chip replicates were used, results from these pooled samples represent mean changes between the replicates.

\section{Rosetta resolver}

This procedure was performed as previously described (Kim et al., 2006). Images and GEML (gene expression markup language) files, including error and $P$-values, were exported from the Agilent Feature Extraction software and deposited into Rosetta Resolver (version 4.0, build 4.0.1.0.7.RSPLIT) (Rosetta Biosoftware, Kirkland, WA). Ratio profiles were combined and intensity plots were generated for each ratio experiment. "Signature genes" were identified, as those having a $P$-value $\leq 0.001$ with a fold-change $\geq 1.5$ in all three biological replicates.

\section{Ingenuity pathways analysis (IPA)}

Signature genes were further analyzed with IPA software (Ingenuity Systems, Redwood City, CA; http://www.ingenuity. com), a web-delivered application that makes use of the Ingenuity Pathways Knowledge Base (IPKB) containing large amounts of individually modeled relationships between gene objects (e.g., genes, mRNAs, and proteins). The procedure was performed as previously described (Liu et al., 2006). Briefly, we uploaded a tab-delimited text file with gene identifiers and their corresponding expression values such as fold-change and $p$-value. Each gene identifier was mapped to its corresponding gene object in the Ingenuity Pathways Knowledge Base. A fold change cutoff of 1.5 -fold and $P<0.001$ was set to identify genes whose expression was differentially regulated. These genes, called "focus genes," were then used as the starting point for generating biological networks. To start building net- works, the application queries the Ingenuity Pathways Knowledge Base for interactions between focus genes and all other gene objects stored in the knowledge base, then generates a set of networks with a network size of approximately 35 genes or proteins. Ingenuity Pathways Analysis then computes a score for each network according to the fit of the user's set of significant genes. The score is derived from a $P$-value and indicates the likelihood of the focus genes in a network being found together because of random chance. A score of 2 indicates a 1 in 100 change that the focus genes are together in a network because of random chance. Therefore, scores of 2 or higher have at least a $99 \%$ confidence level of not being generated by random chance alone. Biological functions are then calculated and assigned to each network.

\section{Real-time RT-PCR analysis}

Real-time RT-PCR analysis was used to quantify the levels of expression of the selected genes (Supplemental Data Table S1). The forward and reverse primers for selected genes were designed using $A B I$ Primer Express software (Applied Biosystems, Foster City, CA). Total RNA was prepared as above and reverse transcribed with MuLV reverse transcriptase and oligo-dT primers (Applied Biosystems), then subjected to an ABI Prism 7900 Sequence Detection System using SYBR green PCR master mix (Applied Biosystems, Cheshire, UK), according to the manufacturer's recommendations (Applied Biosystems). The threshold cycle (CT) values of the real-time PCR were used in the determination of the relative differences in expression between groups. To quantify the gene transcripts, we monitored GAPDH, which served as the internal quantitative control. Each sample was normalized by quantification against its GAPDH content. To determine the normalized value, $2^{-(\triangle \triangle C T)}$ values were compared between control and genistein treated groups, where the changes in crossing threshold $(\Delta \mathrm{CT})=\mathrm{Ct}_{\text {Target gene }}-\mathrm{Ct}_{\mathrm{GAPDH}} \mathrm{RNA}$, and $\Delta \Delta \mathrm{CT}=$ $\Delta \mathrm{Ct}^{\text {control }}-\Delta \mathrm{Ct} \mathrm{t}^{\text {target }}$ as outlined in the Applied Biosystems protocol, and the results are presented as fold change above control.

\section{Western blotting analysis}

Total protein was extracted from treated cells and tissue samples as previously described in our lab (Dixon et al., 2002; Swartz et al., 2005). Then protein was separated on a $4-12 \%$ Nu-Page Bis-Tris (Invitrogen) gel, transferred to a $0.45 \mu \mathrm{M}$ PVDF membrane and probed with a monoclonal anti- human/mouse/rat activin A (1: 500; MAB3381, R\&D Systems Inc., Minneapolis, MN) and a Smad3 (1: 1000 diIution; Sc-8332, Santa Cruz Biotechnology, Santa Cruz, CA). Recombinant human/mouse/rat activin A (338-AC, R\&D Systems Inc.), was used as positive control. Signals were developed by using ECL Western Blotting Analysis System (Amersham pharmacia, Piscataway, NJ) according to the manufacturer's protocol. A densitometer (Fluor ChemTM8900, Alpha Innotech, San Leandro, CA) was used for quantitation of the band intensities. 


\section{Cell proliferation assay}

UtLM cells were seeded into 96-well plates at an initial density of $4 \times 10^{3}$ per well in $100 \mu$ l Eagle's MEM (Invitrogen) supplemented with vitamins, amino acids and $19 \%$ FBS, and then incubated with DMEM/F-12, phenol red-free with charcoal dextran treated FBS for $24 \mathrm{~h}$. The UtLM cells were then cultured with vehicle control $(0.3 \%$ DMSO), genistein $(50 \mu \mathrm{g} / \mathrm{ml})$ alone or genistein in combination with activin $A(20 \mathrm{nM})$ or activin A alone for $96 \mathrm{~h}$. Cell proliferation was measured by spectrophotometry using CellTiter $96^{\mathrm{R}} \mathrm{AQ} \mathrm{u}^{\text {eous }}$ One Solution Cell Proliferation assay (Promega Corporation, Madison, WI) according to the manufacturer's protocol. All conditions were tested in five replicates in triplicate experiments.

\section{Cell cycle analysis}

Flow cytometry was used to determine the percentage of cells in various phases of the cell cycle. The DNA content was determined using propidium iodide $(\mathrm{PI})$ and ethanol fixation. Briefly, UtLM cells were treated with $0 \mu \mathrm{g} / \mathrm{ml}$ (DMSO; control), $50 \mu \mathrm{g} / \mathrm{ml}$ genistein (Sigma), $20 \mathrm{nM}$ activin A (R \& D Systems), or a combination of $50 \mu \mathrm{g} / \mathrm{ml}$ genistein and $20 \mathrm{nM}$ activin A, and incubated in culture for $48 \mathrm{~h}$. The UtLM cells were pelleted and fixed by the slow addition of cold $70 \%$ ethanol to a volume of approximately $2-3 \mathrm{ml}$ with agitation. The volume was adjusted to $5 \mathrm{ml}$ with cold $70 \%$ ethanol and the cells were stored at $-20^{\circ} \mathrm{C}$ overnight. Fixed cells were pelleted from the ethanol, washed once in $3 \mathrm{ml}$ of $1 X$ PBS and stained in $1 \mathrm{ml}$ of $20 \mathrm{ng} / \mathrm{ml}$ propidium iodide, 1,000 units RNaseOne (Promega) in 1X PBS. Analysis was done using a Becton Dickinson FACSort flow cytometer and CELLQuest software (Becton Dickinson Immunocytometry Systems, San Jose, CA). Individual cells (7,500 per experimental sample) were selected by gating on a $\mathrm{PI}$ area versus width dot plot to exclude cell aggregates and debris. Cells were excited using a $488 \mathrm{~nm}$ argon laser and emission was detected at $585 \mathrm{~nm}$. Data was analyzed using Modfit software for Mac version 2.0.

\section{Statistical analysis}

The results are expressed as means \pm S.E. (standard error). The gene expression data of quantitative RT-PCR experiments were analyzed for statistical significance by a Student's $t$-test for two-tailed unpaired comparisons. Two-sided Mann-Whitney test was performed to determine the statistical significance of the results of protein expression and cell proliferation assay between different groups. A probability level of $P$-value $\leq 0.05$ was considered significant.

\section{Supplemental data}

Supplemental data include a table and can be found with this article online at http://e-emm.or.kr/article/article_files/ SP-44-4-04.pdf.

\section{Acknowledgements}

The authors would like to thank Ms. Maria Sifre for her ex- pert assistance with the flow cytometry studies and acknowledge the editorial assistance of the National Institutes of Health (NIH) Fellows Editorial Board. This research was supported by the National Toxicology Program (NTP) and the Intramural Research Program of the NIH, National Institute of Environmental Health Sciences (NIEHS). This article may be the work product of an employee or group of employees of the NIEHS, NTP, NIH, however, the statements, opinions or conclusions contained therein do not necessarily represent the statements, opinions or conclusions of NIEHS, NTP, NIH or the United States government.

\section{References}

Abe Y, Minegishi T, Leung PC. Activin receptor signaling. Growth Factors 2004;22:105-10

Burdette JE, Jeruss JS, Kurley SJ, Lee EJ, Woodruff TK. Activin A mediates growth inhibition and cell cycle arrest through Smads in human breast cancer cells. Cancer Res 2005;65:7968-75

Chegini N, Ma C, Tang XM, Williams RS. Effects of GnRH analogues, 'add-back' steroid therapy, antiestrogen and antiprogestins on leiomyoma and myometrial smooth muscle cell growth and transforming growth factor-beta expression. Mol Hum Reprod 2002;8:1071-8

Chegini N, Luo X, Ding L, Ripley D. The expression of Smads and transforming growth factor beta receptors in leiomyoma and myometrium and the effect of gonadotropin releasing hormone analogue therapy. Mol Cell Endocrinol 2003;209: 9-16

Chwalisz K, Perez MC, Demanno D, Winkel C, Schubert G, Elger W. Selective progesterone receptor modulator development and use in the treatment of leiomyomata and endometriosis. Endocr Rev 2005;26:423-38

Dixon D, Flake GP, Moore AB, He H, Haseman JK, Risinger JI, Lancaster JM, Berchuck A, Barrett JC, Robboy SJ. Cell proliferation and apoptosis in human uterine leiomyomas and myometria.Virchows Arch 2002;441:53-62

Gupta SC, Kim JH, Prasad S, Aggarwal BB. Regulation of survival, proliferation, invasion, angiogenesis, and metastasis of tumor cells through modulation of inflammatory pathways by nutraceuticals. Cancer Metastasis Rev 2010;29:405-34

Harrison CA, Gray PC, Vale WW, Robertson DM. Antagonists of activin signaling: mechanisms and potential biological applications. Trends Endocrinol Metab 2005;16:73-8

Hogg K, Etherington SL, Young JM, McNeilly AS, Duncan WC. Inhibitor of differentiation (Id) genes are expressed in the steroidogenic cells of the ovine ovary and are differentially regulated by members of the transforming growth factor-beta family. Endocrinology 2010;151:1247-56

Jamadar-Shroff V, Papich MG, Suter SE. Soy-derived isoflavones inhibit the growth of canine lymphoid cell lines. Clin Cancer Res 2009;15:1269-76

Kim Y, Ton TV, DeAngelo AB, Morgan K, Devereux TR, Anna C, Collins JB, Paules RS, Crosby LM, Sills RC. Major 
carcinogenic pathways identified by gene expression analysis of peritoneal mesotheliomas following chemical treatment in F344 rats. Toxicol Appl Pharmacol 2006;214: 144-51

Lavigne JA, Takahashi Y, Chandramouli GV, Liu H, Perkins SN, Hursting SD, Wang TT. Concentration-dependent effects of genistein on global gene expression in MCF-7 breast cancer cells: an oligo microarray study. Breast Cancer Res Treat 2008;110:85-98

Lee WY, Huang SC, Tzeng CC, Chang TL, Hsu KF. Alterations of metastasis-related genes identified using an oligonucleotide microarray of genistein-treated HCC1395 breast cancer cells. Nutr Cancer 2007;58:239-46

Leppert PC, Catherino WH, Segars JH. A new hypothesis about the origin of uterine fibroids based on gene expression profiling with microarrays. Am J Obstet Gynecol 2006;195: 415-20

Levens E, Luo X, Ding L, Williams RS, Chegini N. Fibromodulin is expressed in leiomyoma and myometrium and regulated by gonadotropin-releasing hormone analogue therapy and TGF-beta through Smad and MAPK-mediated signalling. Mol Hum Reprod 2005;11:489-94

Litovkin KV, Ivanova OV, Bauer A, Hoheisel JD, Bubnov VV, Zaporozhan VN. Microarray study of gene expression in uterine leiomyoma. Exp Oncol 2008;30:106-11

Liu J, Xie Y, Ducharme DM, Shen J, Diwan BA, Merrick BA, Grissom SF, Tucker CJ, Paules RS, Tennant R, Waalkes MP. Global gene expression associated with hepatocarcinogenesis in adult male mice induced by in utero arsenic exposure. Environ Health Perspect 2006;114:404-11

Liu J, Matsuo H, Xu Q, Chen W, Wang J, Maruo T. Concentration-dependent effects of a selective estrogen receptor modulator raloxifene on proliferation and apoptosis in human uterine leiomyoma cells cultured in vitro. Hum Reprod 2007;22:1253-9

Luo X, Ding L, Xu J, Chegini N. Gene expression profiling of leiomyoma and myometrial smooth muscle cells in response to transforming growth factor-beta. Endocrinology 2005; 146:1097-118

McLaughlin M, Bromfield JJ, Albertini DF, Telfer EE. Activin promotes follicular integrity and oogenesis in cultured pre-antral bovine follicles.Mol Hum Reprod 2010;16:644-53

Moore AB, Castro L, Yu L, Zheng X, Di X, Sifre MI, Kissling GE, Newbold RR, Bortner CD, Dixon D. Stimulatory and inhibitory effects of genistein on human uterine leiomyoma cell proliferation are influenced by the concentration. Hum Reprod 2007;22:2623-31
Mukhopadhyay A, Chan SY, Lim IJ, Phillips DJ, Phan TT. The role of the activin system in keloid pathogenesis. Am J Physiol Cell Physiol 2007;292:C1331-8

Pan Q, Luo X, Chegini N. Genomic and proteomic profiling I: leiomyomas in African Americans and Caucasians. Reprod Biol Endocrinol 2007;5:34

Pavese JM, Farmer RL, Bergan RC. Inhibition of cancer cell invasion and metastasis by genistein. Cancer Metastasis Rev 2010;29:465-82

Piao M, Mori D, Satoh T, Sugita Y, Tokunaga O. Inhibition of endothelial cell proliferation, in vitro angiogenesis, and the down-regulation of cell adhesion-related genes by genistein. Combined with a cDNA microarray analysis. Endothelium 2006;13:249-66

Polkowski K, Mazurek AP. Biological properties of genistein. A review of in vitro and in vivo data. Acta Pol Pharm 2000; 57:135-55

Rackow BW, Arici A. Options for medical treatment of myomas. Obstet Gynecol Clin North Am 2006;33:97-113

Swartz CD, Afshari CA, Yu L, Hall KE, Dixon D. Estrogeninduced changes in IGF-I, Myb family and MAP kinase pathway genes in human uterine leiomyoma and normal uterine smooth muscle cell lines. Mol Hum Reprod 2005;11: 441-50

Ten Dijke P, Hill CS. New insights into TGF-beta-Smad signalling. Trends Biochem Sci 2004;29:265-73

Tulandi T. Treatment of uterine fibroids--is surgery obsolete? N Engl J Med 2007;356:411-3

Viswanathan M, Hartmann K, McKoy N, Stuart G, Rankins $\mathrm{N}$, Thieda P, Lux LJ, Lohr KN. Management of uterine fibroids: an update of the evidence. Evid Rep Technol Assess (Full Rep) $2007 ; 1-122$

Walker CL, Stewart EA. Uterine fibroids: the elephant in the room. Science 2005;308:1589-92

Ye R, Goodarzi AA, Kurz EU, Saito S, Higashimoto Y, Lavin MF, Appella E, Anderson CW, Lees-Miller SP. The isoflavonoids genistein and quercetin activate different stress signaling pathways as shown by analysis of site-specific phosphorylation of ATM, p53 and histone H2AX. DNA Repair (Amst) 2004;3:235-44

Zhao Y, Wen Y, Polan ML, Qiao J, Chen BH. Increased expression of latent TGF-beta binding protein-1 and fibrillin-1 in human uterine leiomyomata. Mol Hum Reprod 2007;13:343-9 